\title{
El fenómeno de sexting en adolescentes, Cuenca-Ecuador
}

\section{The sexting phenomenon in adolescents, Cuenca-Ecuador}

\author{
María Magdalena Piedra Argudo ${ }^{1}$, Jeanneth Tapia Cárdenas*2, José Luis Once Campoverde ${ }^{2}$, Zoila Katherine \\ Salazar Torres $^{2}$, Vinicio Marcelo Tenemaza Ruilova ${ }^{2}$ \\ ${ }^{1}$ Hospital Monte Sinai, Cuenca, Ecuador \\ ${ }^{2}$ Universidad Católica de Cuenca, Ecuador \\ *janethtapiac@hotmail.com
}

DOI: https://doi.org/10.26871/killkanasalud.v4i4.750

\begin{abstract}
Resumen
Antecedentes: el sexting es el envío de contenidos de tipo sexual, realizadas por el propio remitente y que son enviadas a otras personas. El sexting puede involucrar problemas legales e incluso el CiberBullying, ciberacoso, o sextorsion. Objetivo: determinar la frecuencia y caracterizar el sexting en los adolescentes de la Unidad Educativa del Milenio Manuela Garaicoa de Calderón, Cuenca 2018. Materiales y métodos: se realizó un estudio observacional, cuantitativo, descriptivo, se aplicó una encuesta a 268 adolescentes matriculados en la institución participante para el período lectivo 2017-2018, los cuales fueron seleccionados aleatoriamente, se indagó sobre características sociodemográficas, frecuencia y caracterización de sexting. Resultados: el promedio de edad fue 15,86 años, el grupo etario más frecuente se ubicó entre los 14 a 16 años, 67,9\% de sexo femenino, 69,4\% residía en áreas urbanas. El 63,1 \% afirmó practicar sexting; 58,2 \% fue pasivo y $20,5 \%$ activo. Sobre sexting pasivo, el tipo de contenido más frecuente fueron las fotos $77,6 \%$, siendo Facebook la red social más usada 78,8\%. En el sexting activo su práctica se dio de manera voluntaria en $81,8 \%$ y se caracterizó por el envío de textos con proposiciones sobre actividad sexual mediante un móvil en $41,8 \%$. Conclusiones: la práctica de sexting en los adolescentes fue elevada, siendo el sexting pasivo dos veces más frecuente que el activo.
\end{abstract}

Palabras clave: adolescencia, prevalencia, ciberacoso, sexualidad, conducta sexual.

\begin{abstract}
Background: sexting is the sending of sexual content, made by the sender himself and sent to other people. Sexting can involve legal problems and even cyberBullying, cyberBullying, or sextorsion. Objective: to determine the frequency and characterize sexting in adolescents of the Millennium Educational Unit Manuela Garaicoa de Calderón, Cuenca 2018. Materials and methods: an observational, quantitative, descriptive study was carried out, a survey was applied to 268 adolescents enrolled in the participating institution for the 2017-2018 school period, which were randomly selected, it was inquired about sociodemographic characteristics, frequency and characterization of sexting. Results: the average age was 15.86 years, the most frequent age group was between 14 to 16 years, $67.9 \%$ female, 69.4\% lived in urban areas. $63.1 \%$ affirmed to practice sexting; $58.2 \%$ was passive and $20.5 \%$ active. Regarding passive sexting, the most frequent type of content was photos, 77.6\%, with Facebook being the most used social network 78.8\%. In active sexting, their practice was voluntary in $81.8 \%$ and it was characterized by sending texts with proposals about sexual activity through a mobile phone in 41.8\%. Conclusions: the practice of sexting in adolescents was high, with passive sexting being twice more frequent than active.
\end{abstract}

Keywords: adolescence, prevalence, cyber Bullying, sexuality, sexual behavior.

\section{Introducción}

Actualmente los avances sociales se desarrollan simultáneamente con los progresos tecnológicos y de comunicación, los cuales han facilitado enormemente las relaciones interpersonales, por ejemplo, los teléfonos móviles han conseguido una tasa de penetración impresionante de casi la totalidad de la humanidad. ${ }^{1}$ Sin embargo, es importante recalcar que con el advenimiento de novedosas tecnologías han nacido también nuevos delitos, lo que nos lleva a pensar de no ser utilizadas responsablemente pueden transformar- se en un arma peligrosa para los usuarios, especialmente los adolescentes. $^{2}$

El problema del uso de internet y de los dispositivos móviles para compartir información entre los adolescentes, radica en el hecho de que se encuentran en una etapa en la cual su criterio y personalidad aún no se ha formado, volviéndoles un blanco fácil para ser atrapados por tendencias peligrosas. ${ }^{3}$

En este sentido, el concepto de sexting surge de la unión de dos vocablos, sex (sexo) y texting (envío de mensajes 
de texto), no obstante, este término se ha ampliado, con una definición que abarca otros aspectos como: el envío o reenvío de contenidos de orden erótico y/o sexual, que impliquen fotos, videos, textos a otras personas a través de dispositivos tecnológicos o medios virtuales. ${ }^{1,3,4}$

Es por ello que el uso inadecuado de internet supone riesgos para los jóvenes entre los cuales podemos citar: conductas sexuales peligrosas, pornografía infantil, mayor probabilidad de victimización online, entre otras. ${ }^{5}$ Se cree que la expansión de dispositivos móviles que permitan el acceso a internet significa un completo desafío dentro de la sociedad actual, ${ }^{6,7}$ principalmente en países desarrollados donde las políticas y leyes, así como la percepción en general de la sociedad permite ampliamente la aparición de tendencias dañinas para los adolescentes que no solo permanecen en sus países de origen, sino que vencen fronteras convirtiéndose en amenazas para la humanidad. ${ }^{2}$

En base a lo expuesto, resulta indispensable despertar el interés por determinar que fenómenos están presentes entre los jóvenes, quienes actualmente viven y se desarrollan en un medio en el que la tecnología ha superado todas las expectativas y nos ha invadido de tal manera que pueden llegar a ser sujetos de abusos y delitos que atenten contra su integridad. ${ }^{1}$

En la presente investigación se buscó poner en evidencia la prevalencia y las características de una nueva problemática que nace a raíz del internet como lo es el sexting que, si bien no ha sido analizada a profundidad y que seguramente resulta extraña para la mayoría de las personas, incluso para los mismos jóvenes que la están experimentando, podría estar presente entre nosotros amenazando profundamente el bienestar de la población adolescente.

\section{Marco teórico}

El termino sexting resulta de la combinación de dos palabras en el idioma inglés que son sex = sexo y texting $=$ mensaje de texto, dicha palabra nace por primera vez en Reino Unido alrededor del $2005 .{ }^{8}$ Hace referencia a un fenómeno relativamente nuevo, que consiste en el envío de mensajes, videos o fotos con contenido sexual y/o erótico. ${ }^{9}$ En primera instancia se reconocía como sexting única y exclusivamente a la emisión de mensajes con contenido sexual, sin embargo, con el advenimiento de los smartphone se ha llegado a la conclusión de que dicho fenómeno puede estar representado no solo por el envío de contenidos de este tipo, sino también la recepción de los mismos, y la transacción electrónica de fotos, videos y textos sexualmente explícitos o sugerentes. ${ }^{10}$

Reyns, manifiesta que el sexting hace referencia al envío de mensajes o fotografías sexualmente explícitos desde un teléfono móvil a otro, y también por medio del correo electrónico o la mensajería instantánea. ${ }^{11}$

McLaughlin lo define como "la producción de textos, imágenes o vídeos digitales en los que aparecen menores de edad o personas adultas estando desnudos/as o semidesnudos/as, con contenidos sexuales y captados por sí mismos/as, así como su transmisión a otras personas, a través de los medios tecnológicos como la telefonía móvil, el correo electrónico o incluso las redes sociales". ${ }^{12}$

El sexting es entonces el fenómeno por el cual un individuo realiza el envío o recibe mensajes con contenido sexual o erótico sobre sí mismo o sobre terceros; dichos mensajes pueden contener imágenes, texto o videos, y los mismos pueden ser enviados o recibidos por cualquier dispositivo tecnológico. ${ }^{13-15}$

Las principales particularidades que caracterizan a la práctica de sexting son:

- El uso de los medios tecnológicos: se ha identificado como facilitadores de estas prácticas a los teléfonos móviles, ordenadores, tabletas y redes sociales como: Facebook, Twitter, Tinder, Instagram, Snapchat y a los medios de mensajería instantánea como WhatsApp. ${ }^{16-19}$

- Carácter sexual y/o erótico de los contenidos: se ha de entender como contenido de carácter sexual a aquellos que hacen referencia a comportamientos sexuales implícitos con el propio sujeto o con otras personas; mientras que el contenido erótico ha de definirse como situaciones o actos sugerentes de actividad sexual. ${ }^{16-19}$

- Origen: hace referencia a que la producción o práctica de dicho fenómeno puede ser propia o ajena. ${ }^{16-19}$

- Edad: es muy importante determinar la edad en la que se tiende a caer en la práctica de sexting, pudiendo afirmar la relación directa entre el incremento de la misma con los años de vida. ${ }^{16-19}$

- Sexo: se ha visto que dicho fenómeno tiende a ser mayormente practicado por mujeres, sin embargo, se considera también que no existen diferencias significativas en cuanto al sexo de quienes lo practican. ${ }^{16-19}$

- Voluntariedad: el envío de este tipo de contenidos puede realizarse de forma voluntaria o también puede hacérselo de manera obligada. ${ }^{16-19}$ Esta última característica se refiere a las influencias y presiones sociales que influyen de una manera desmesurada en la creación de este tipo de contenidos, y que también resulta importante sobre todo entre los adolescentes para quienes el círculo social o la pareja representa un pilar fundamental en esta etapa de sus vidas. ${ }^{16-19}$

Se ha realizado una subdivisión del fenómeno de sexting, de acuerdo a la forma en que este se manifiesta, el tipo de contenido enviado, o el uso que se le da a dichos mensajes. De manera general el sexting puede ser activo cuando la persona envia fotos o contenidos de si mismo en posturas provocativas o inapopiadas y pasivo si por el contrario, se recibe contenidos eroticos, según esta clasificacion se habla entonces de un emisor y un receptor de sexting, ${ }^{20,21}$

Una vez identificada la práctica de sexting, se han planteado serias consecuencias asociadas a la presentecia latente de este fenómeno siendo las más comunes:

- Bullying y CiberBullying: se trata del maltrato entre iguales, implica conductas hostiles por parte de un individuo o grupo en el entorno online. Dentro del sexting 
este tipo de violencia puede evidenciarse en acciones como: insultos o difusión de rumores, hostigamiento, denigración y el "morphing" que consiste en usar la fotografía de la víctima montando su rostro en el cuerpo de otra persona, con el propósito de ridiculizarla. ${ }^{21}$

- Ciberstalking: hace referencia al acoso cibernético, consiste en la invasión de la vida de una persona, en este caso protagonista de sexting, que de manera repetitiva y sin consentimiento es acosada y perseguida a través de las tecnologías de internet. ${ }^{21}$

- Sextorsión: es una forma de explotación sexual, en la cual se continúa solicitando envío de imágenes con contenidos sexuales y/o eróticos a la víctima, a cambio de no hacer público imágenes, videos o mensajes enviados con anterioridad. Puede llegarse incluso a solicitar la práctica de relaciones sexuales. ${ }^{21}$

- Grooming: término utilizado para definir a los abusadores de menores. Consiste en que una persona adulta establece contacto con un menor de edad y finge establecer fuertes lazos de amistad, con el fin de conseguir contacto sexual online, o práctica de sexting con el/la adolescente. $^{21}$

- Teen Dating Violence: es un nuevo acto de violencia que surge a partir de esta práctica, que consiste en el maltrato dentro de las relaciones entre adolescentes. En este caso se obliga a la pareja ya sea a la introducción al sexting, o a continuar enviando contenidos sexuales o eróticos con amenazas de terminar la relación o hacer públicos contenidos ya enviados. ${ }^{20-22}$

\section{Metodología}

Se trató de un estudio cuantitativo, observacional, descriptivo. El universo estuvo constituido por 797 estudiantes matriculados en el período lectivo 2017-2018, mismos que se encontraban cursando el octavo de básica al tercero de bachillerato de la Unidad Educativa del Milenio Manuela Garaicoa de Calderón en su sección vespertina. Se calculó una muestra de 268 estudiantes y se realizó un método de muestreo estratificado basado en el número de estudiantes de los diferentes cursos de la población del estudio, además se procedió a realizar una aleatorización sistemática de la muestra de acuerdo al listado de los alumnos para dar mayor representatividad a la misma. Como técnica de recolección aplicó una encuesta estructurada anónima, previa la firma del consentimiento y/o asentimiento informado tanto de los representantes legales así como los participantes.

El instrumento que se utilizó para evaluar el sexting fue un formulario diseñado y validado en otra investigación, ${ }^{23}$ mismo que fue sometido a un plan piloto con 20 estudiantes de la Unidad Educativa Zoila Aurora Palacios, llegando al instrumento final de la presente investigación.
Tabla 1: Distribución según características sociodemográficas de 268 adolescentes de la Unidad Educativa del Milenio Manuela Garaicoa de Calderón, Cuenca 2018

\begin{tabular}{lcc}
\hline & Frecuencia & Porcentaje \\
\hline Edad & & \\
\hline $10-13$ años & 24 & 8,9 \\
$14-16$ años & 131 & 48,9 \\
$17-19$ años & 113 & 42,2 \\
Total & 268 & 100 \\
\hline Sexo & & \\
\hline Hombre & 86 & 32,1 \\
Mujer & 182 & 67,9 \\
Total & 268 & 100 \\
\hline Residencia & & \\
\hline Urbano & 186 & 69,4 \\
Rural & 82 & 30,6 \\
Total & 268 & 100 \\
\hline Nivel de instrucción & & \\
\hline Educación básica & 37 & 13,8 \\
Bachillerato & 231 & 86,2 \\
Total & 268 & 100 \\
\hline
\end{tabular}

Participaron 268 estudiantes, el 48,9\% de adolescentes tuvieron rangos etarios entre 14 y 16 años, siendo el promedio de edad 15,86 con una desviación estándar de 1,534. El $67,9 \%$ de encuestados correspondió a mujeres, un 69,4\% residían en las áreas urbanas y 86,2\% pertenecían a nivel educativo de bachillerato (tabla 1)

Tabla 2: Distribución según características sociodemográficas de 268 adolescentes de la Unidad Educativa del Milenio Manuela Garaicoa de Calderón, Cuenca 2018

\begin{tabular}{lcc}
\hline & Frecuencia & Porcentaje \\
\hline sexting & & \\
\hline $\mathrm{Si}$ & 169 & 63,1 \\
$\mathrm{No}$ & 99 & 36,9 \\
Total & 268 & 100 \\
\hline sexting pasivo & & \\
\hline $\mathrm{Si}$ & 156 & 58,2 \\
$\mathrm{No}$ & 112 & 41,8 \\
Total & 268 & 100 \\
\hline sexting activo & & \\
\hline Si & 55 & 20,5 \\
No & 213 & 79,5 \\
Total & 268 & 100 \\
\hline
\end{tabular}

El 63,1\% de adolescentes participantes en el estudio practicó sexting, con una frecuencia de sexting pasivo en un $58.2 \%$ y el activo en $20,5 \%$ (tabla 2 ). 
Tabla 3: Caracterización de sexting de acuerdo a: etapa de la adolescencia, sexo, residencia y nivel de instrucción, en la Unidad Educativa del Milenio Manuela Garaicoa de Calderón, Cuenca 2018

\begin{tabular}{lcc}
\hline & \multicolumn{2}{c}{ sexting } \\
\cline { 2 - 3 } & Frecuencia & Porcentaje \\
\hline Etapas de la adolescencia & & \\
\hline Temprana (10-13 años) & 10 & 5,9 \\
Media (14-16 años) & 86 & 50,9 \\
Tardía (17-19 años) & 73 & 43,2 \\
Total & 169 & 100 \\
Sexo & & \\
\hline Hombre & 63 & 37,3 \\
Mujer & 106 & 62,7 \\
Total & 169 & 100 \\
\hline Residencia & & \\
\hline Urbana & 116 & 68,6 \\
Rural & 53 & 31,4 \\
Total & 169 & 100 \\
\hline Nivel de instrucción & \\
\hline Básica & 16 & 9,5 \\
Bachillerato & 153 & 90,5 \\
Total & 169 & 100 \\
\hline
\end{tabular}

Además, se observó que la práctica de sexting prevaleció en el rango de edad de 14 a 16 años, correspondiente a la etapa media de la adolescencia 50,9\%. En cuanto al sexo, existió mayor práctica en las mujeres $62,7 \%$. La frecuencia de sexting resultó ser mayor en el área urbana 68,6\% y fue el bachillerato donde más se manifestó este fenómeno $90,5 \%$ (tabla 3 ).

Tabla 4: Caracterización de sexting pasivo en los adolescentes de la Unidad Educativa del Milenio Manuela Garaicoa de Calderón, Cuenca 2018

\begin{tabular}{lcc}
\hline & Frecuencia & Porcentaje \\
\hline Tipo de contenido & & \\
\hline Mensajes de texto & 76 & 48,7 \\
Fotos & 121 & 77,6 \\
Videos & 57 & 36,5 \\
Otros & 3 & 4,5 \\
\hline Medio & & \\
\hline WhatsApp & 83 & 53,2 \\
Facebook & 123 & 78,8 \\
Twitter & 1 & 0,6 \\
Instagram & 28 & 17,9 \\
Snapchat & 7 & 4,5 \\
Tinder & 2 & 1,3 \\
Otros & 16 & 10,3 \\
\hline
\end{tabular}

El principal tipo de contenido sexual y/o erótico recibido fueron las fotografías en un $77,6 \%$, seguido de mensajes de texto $48,7 \%$; además el principal medio por el cual los adolescentes practicaron sexting pasivo fue Facebook en un $78,8 \%$, seguido de WhatsApp en un $53.2 \%$ (tabla 4).

Tabla 5: Caracterización, voluntariedad y consecuencias de la práctica de sexting Activo en los adolescentes de la Unidad Educativa del Milenio Manuela Garaicoa de Calderón, Cuenca 2018

\begin{tabular}{llcc}
\hline Caracterización & & Frecuencia & Porcentaje \\
\hline & Nunca & 39 & 70,9 \\
Foto y/o video sexualmente sugerente & Casi nunca & 8 & 14,5 \\
& En ocasiones & 8 & 14,5 \\
& Total & 55 & 100 \\
\hline \multirow{2}{*}{ Foto y/o video en ropa interior por teléfono móvil } & Nunca & 38 & 69,1 \\
& Casi nunca & 12 & 21,8 \\
& En ocasiones & 4 & 7,3 \\
& Frecuentemente & 1 & 1,8 \\
Foto y/o video en ropa interior por redes sociales & Total & 55 & 100 \\
\hline & Nunca & 46 & 83,6 \\
& Casi nunca & 6 & 10,9 \\
& En ocasiones & 3 & 5,5 \\
Foto y/o video desnudo por teléfono móvil & Total & 55 & 100 \\
\hline & Nunca & 45 & 81,8 \\
& Casi nunca & 5 & 9,1 \\
& En ocasiones & 4 & 7,3 \\
\hline
\end{tabular}


Tabla 5: Caracterización, voluntariedad y consecuencias de la práctica de sexting Activo en los adolescentes de la Unidad Educativa del Milenio Manuela Garaicoa de Calderón, Cuenca 2018...(continuación)

\begin{tabular}{llcc}
\hline Caracterización & & Frecuencia & Porcentaje \\
\hline \multirow{2}{*}{ Foto y/o video desnudo por redes sociales } & Nunca & 52 & 94,5 \\
& Casi nunca & 2 & 3,6 \\
& En ocasiones & 1 & 1,8 \\
& Total & 55 & 100 \\
\hline Texto sexualmente sugerente por teléfono móvil & Nunca & 21 & 38,2 \\
& Casi nunca & 17 & 30,9 \\
& En ocasiones & 13 & 23,6 \\
& Frecuentemente & 4 & 7,3 \\
Texto sexualmente sugerente por redes sociales & Total & 55 & 100 \\
& Nunca & 40 & 72,7 \\
& Casi nunca & 6 & 10,9 \\
& En ocasiones & 9 & 16,4 \\
Texto con proposiciones sobre actividad sexual por teléfono & Total & 55 & 100 \\
móvil & Nunca & 16 & 29,1 \\
& Casi nunca & 23 & 41,8 \\
& En ocasiones & 13 & 23,6 \\
& Frecuentemente & 3 & 5,5 \\
Texto con proposiciones sobre actividad sexual por redes & Total & 55 & 100 \\
sociales & Nunca & 40 & 72,7 \\
& Casi nunca & 9 & 16,4 \\
& En ocasiones & 5 & 9,1 \\
& Frecuentemente & 1 & 1,8 \\
& Total & 55 & 100 \\
\hline
\end{tabular}

Tabla 6: Voluntariedad y consecuencias de la práctica de sexting Activo en los adolescentes de la Unidad Educativa del Milenio Manuela Garaicoa de Calderón, Cuenca 2018

\begin{tabular}{lcc}
\hline & Frecuencia & Porcentaje \\
\hline Voluntariedad & & \\
\hline $\mathrm{Si}$ & 45 & 81,8 \\
$\mathrm{No}$ & 10 & 18,2 \\
\hline Consecuencias & & \\
\hline $\mathrm{Si}$ & 25 & 45,5 \\
$\mathrm{No}$ & 30 & 54,5 \\
\hline Tipo de consecuencias & & \\
\hline Bullying y CiberBullying & 4 & 7,3 \\
Ciberstalking & 9 & 16,4 \\
Sextorsión & 2 & 3,6 \\
Grooming & 16 & 29,1 \\
Teen Dating Violence & 7 & 12,7 \\
\hline
\end{tabular}

La práctica del sexting activo se fundamentó en el envío de fotos y/o videos en ropa interior a través de un teléfono móvil en un $21,8 \%$ de la población y también en el envío de mensajes de texto con proposiciones sobre actividad sexual mediante un teléfono móvil $41,8 \%$; ambas con una frecuencia de "casi nunca". El 81,8\% de los adolescentes han practicado sexting activo de manera voluntaria y hemos encontrado que de las 55 personas que han practicado sexting activo, los adolescentes han experimentado consecuencias en un $45,5 \%$, dentro de las cuales la más frecuente resultó ser el Grooming que aparece en un 29,1\% de los jóvenes (tabla 5 y 6 ).

\section{DISCUSIÓN}

La revolución tecnológica que ha impactado el siglo XXI ha acarreado consigo el surgimiento de nuevas tendencias que han transformado las relaciones sociales, afectivas y sexuales, entre las que destaca el sexting; fenómeno mediante el cual los y las jóvenes han encontrado un espacio natural de relación y una nueva forma de interacción sexual, nuestro estudio se centró en conocer la frecuencia y caracterizar el sexting en los adolescentes por su importancia en el ámbito social e inclusive de la salud pública, pero, en especial, debido a los innumerables peligros que dicha práctica conlleva.

La existencia de estudios sobre el tema lamentablemente es escasa, lo que limita el conocimiento sobre su práctica, sus características y sus riesgos, sobre todo en países en vías de desarrollo. En España se han llevado a cabo algunas investigaciones, Gámez-Guadix M, De Santisteban P, Resett S, durante el 2016 analizaron la prevalencia de sexting en un grupo de adolescentes españoles y la posible relación que ello podría tener con su personalidad, encontrando que el envío o recepción de contenidos sexuales entre los jóvenes por medio de internet está presentando una importante atención social. ${ }^{10}$ 
Ecuador, como país, no ha logrado identificar estudios respecto al tema, sin embargo, a nivel local se cuenta con un estudio realizado por Cajamarca M, en la Unidad Educativa Octavio Cordero Palacios en el año 2016 , donde la autora afirma que sus resultados reflejan "un bajo nivel de incidencia de sexting en los adolescentes", pero que sí se logró determinar conductas de riesgo entre los participantes mismos que manifestaron mantener contacto con personas desconocidas a nivel online y que en el caso de enviar fotos, videos u otros, los principales destinatarios serían sus parejas sentimentales o la persona por quien se manifieste un gusto especial y que el aparato principal de comunicación sería un teléfono móvil inteligente.

En el estudio de Alonso P, España, 2017 sobre "Evaluación del fenómeno del sexting y de los Riesgos Emergentes de la Red en adolescentes de la provincia de Ourense" el rango de edad de la población estuvo entre los 12 a 21 años con un promedio de edad de 15,63 años, el 51,1\% pertenecía al sexo femenino, el nivel educativo fue de 32,9\% para el bachillerato y el 50,8\% de la población vivía en el área urbana. De la misma forma, Gámez-Guadix M, De Santisteban P, Resett S, España, 2016, en su investigación sobre "sexting entre adolescentes españoles: prevalencia y asociación con variables de personalidad" trabajaron con jóvenes cuyos rangos de edad estuvieron entre 12 y 17 años, una media de 14,06, el 49,9\% fueron mujeres, 37,6\% correspondían al bachillerato y todos pertenecían al área urbana. A nivel local, Cajamarca M, Cuenca, 2016, en su investigación titulada "Identificación de los niveles de sexting entre adolescentes" trabajó con jóvenes de entre 14 a 19 años, la media de edad fue de 16,1 años, en este caso el $53 \%$ correspondían al sexo masculino, $78.6 \%$ estudiaba en bachillerato y el $57,8 \%$ pertenecían al área urbana. Nuestro estudio, al igual que los citados, se enfocó en la población adolescente con la diferencia de que nosotros abarcamos las tres etapas de la adolescencia, por ello nuestro rango de edad fue de 10 a 19 años, la edad promedio fue de 15 , 86 años, la población se caracterizó por ser mayormente del sexo femenino con un $67,9 \%$, el $69,4 \%$ de los participantes pertenecían al área urbana y el 86,2\% se encontraban cursando el bachillerato, discrepando únicamente en cuanto al sexo según lo encontrado por Miriam Cajamarca. ${ }^{10}$

Sobre los resultados obtenidos en cuanto a la frecuencia y tipo de sexting entre los adolescentes pertenecientes a la institución en un estudio a través de una encuesta que involucró preguntas cerradas sobre la recepción de mensajes con contenido sexual o erótico y una escala de tipo Likert para indagar respecto al envío de estos contenidos, nuestros resultados muestran que la práctica general de sexting se sitúa en un 63,1\%, y que de entre ellos el 58,2\% practicaba sexting pasivo frente a un $20,5 \%$ que practicó sexting activo. Estos resultados difieren de los encontrados en la investigación de Alonso P, España, 2017, donde la frecuencia general de sexting se situó en un $59,5 \%$, teniendo un $45,5 \%$ de sexting pasivo y un $30,6 \%$ de sexting activo, resultando ser la práctica general de sexting y de sexting pasivo inferior a la encontrada en nuestra investigación, pero con una tendencia mucho mayor a la práctica de sexting activo.

En este mismo ámbito, en el trabajo bibliográfico sobre el sexting entre adolescentes españoles de Gámez-Guadix M, De Santisteban P, Resett S, España, 2016, se encontró una prevalencia general de sexting del 13,5\%, estudio en el cual además se determinó que dicha práctica tiende a incrementarse de forma directa a medida que aumenta la edad, pues a los 12 años la frecuencia de sexting se encontraba en 3,4\% y esta ascendió al 36,1\% a los 17 años, de la misma forma una investigación realizada en 20 países de Europa durante el año 2014 por Baumgartner S, determinó que la frecuencia de sexting para ese tiempo en el viejo continente oscilaba entre 1 a $5 \%$ de la población adolescente, mientras que para el 2016 Villacampa C en su estudio titulado "sexting: prevalencia, características personales y conductuales y efectos en una muestra de adolescentes en España" demostró el enorme auge y popularidad que este fenómeno alcanzaba con los años al obtener un resultado de sexting general del $35 \%$, con una práctica de sexting pasivo del $28,6 \%$ y un $8,2 \%$ para el sexting activo, concordando además con Gámez-Guadix M y colaboradores sobre la relación directa existente entre la práctica de sexting y el incremento de la edad. En Estados Unidos, en cambio, Rice E y colaboradores encontraron para el 2014 una presencia de $20 \%$ de sexting pasivo y $5 \%$ de sexting activo entre los adolescentes, con una frecuencia general oscilante entre 2 y $54 \%$ para todo el país. Finalmente, en Latinoamérica se cuenta con datos respecto a México en donde el Instituto Nacional de Transparencia (INAI) manifiesta una frecuencia de sexting pasivo del 36,7 \% y sexting activo del 10,2\%. Dicho esto, podemos afirmar con certeza que los resultados obtenidos en nuestro estudio no se encuentran lejos de una realidad que ha sido al menos demostrada y estudiada a fondo en España, y datos a los que se suman también los obtenidos en Estados Unidos y México demostrando entonces la enorme fama que esta tendencia está ganando entre los jóvenes. ${ }^{10,16,17,21-23}$

Sobre la caracterización del sexting, Fleschler et al, España, 2013, manifiesta que los adolescentes entre 14 a 16 años son los que más practican sexting, datos que concuerdan con los mencionados por Alonso P, en su investigación en España durante el 2017 y que son similares a nuestros resultados pues en la población estudiada hemos encontrado que el grupo adolescente más vinculado con este fenómeno fue el de la adolescencia media (14 a 16 años) con un 50,9\%. Respecto al sexo, Alonso P, identificó al sexo masculino como el más "sexter", lo que difiere de nuestros resultados pues el $62,7 \%$ fueron del sexo femenino. Para Villacampa C, quien realizó un estudio respecto al tema en la ciudad de Cataluña, durante el 2016, el sexo no muestra diferencias significativas, pues en sus resultados la participación femenina en sexting fue del $52 \%$ frente a un $47 \%$ para el sexo opuesto, y manifiesta incluso que no es el sexo el factor determinante para la práctica de este 
fenómeno sino más bien la edad. En cuanto a la localidad Fleschler et al, Alonso P y Villacampa C concuerdan que es el área urbana quien muestra la mayor frecuencia de sexting con un $54,5 \%, 60,3 \%$ y $58,7 \%$ respectivamente, datos que coinciden con los encontrados en este estudio pues el $68,6 \%$ de jóvenes involucrados a esta tendencia manifestaron residir en el área urbana. ${ }^{16}$

En este trabajo de investigación hemos mencionado a su vez una tipificación de sexting en dos subgrupos, sexting pasivo y activo. Para el sexting pasivo hemos encontrado que el principal tipo de contenido visto o recibido por los adolescentes fueron las fotos en un 77,6\%, seguido de los mensajes de texto con el 48,7\%, resultados que difieren de los encontrados por Gámex Guadix, et al, España 2015, en donde el principal tipo de contenido fueron los mensajes de texto con el $58,3 \%$, seguido de las fotos en un $27,8 \%$. Respecto al principal medio usado en la práctica de este fenómeno, nuestros datos revelan que Facebook es el medio más usado $78,8 \%$ seguido de WhatsApp con un 53,2\%, resultados que difieren de los encontrados por Van Outsyel J et al, Bulgaria, 2017 en su investigación sobre percepciones, motivos y consecuencias de las prácticas de sexting, donde Snapchat destaca como el medio más frecuente con un 65,2\%. En cambio, en Cataluña Villacampa C, encontró que, en el 2016, la plataforma más usada para la práctica de esta tendencia fue WhatsApp con un $98 \%$, seguida de Facebook en un $84 \%$, resultados que también se diferencian con los nuestros y cuya variación probablemente dependa del nivel de popularidad que las distintas redes sociales han alcanzado en los diferentes países. ${ }^{12,15,16}$

Sobre sexting activo, voluntariedad y consecuencias de su práctica, son pocos los estudios realizados y datos encontrados; pese a ello en la investigación citada ya con anterioridad, Alonso $\mathrm{P}$ expone que en el caso del sexting activo el 30,6\% de jóvenes españoles manifestaron que al menos alguna vez han enviado fotos y/o videos de sí mismos con contenidos sexualmente sugerentes, mientras que un $48,5 \%$ en cambio afirmó enviar mensajes de texto sexualmente sugerente usando un teléfono móvil, resultados que difieren con los encontrados en la investigación propuesta en la que un $21,8 \%$ admitió que al menos alguna vez practicó el envío de una foto y/o video de sí mismo en ropa interior mediante un teléfono móvil y el $41,8 \%$ admitió el envío de un mensaje de texto con proposiciones sobre actividad sexual usando un teléfono móvil, además nos interesamos en averiguar a su vez si el envío de estos contenidos se lo hizo de manera voluntaria o no, pues en el estudio referente ya mencionado se obtuvo que el $37,9 \%$ de los jóvenes se vieron obligados a enviar contenidos de estas características, en nuestra investigación el resultado fue menor pues solo el $18,2 \%$ se sintieron obligados, resulta preocupante saber que los jóvenes se están exponiendo a grandes peligros en contra de su voluntad, finalmente hemos concluido este estudio con la indagación respecto al padecimiento o no de consecuencias tras el envío de contenidos sexuales o eróticos encontrando que el $45,5 \%$ de los participantes efectivamente sufrieron consecuencias, siendo la más frecuente el Grooming con un 29,1\%, y que difieren de las encontradas en España, 2017, en donde $53,6 \%$ de los jóvenes presentaron consecuencias y entre las cuales la más común resultó ser el CiberBullying con el $40 \%$, aunque las principales consecuencias encontradas en ambos estudios difieran, resulta importante destacar que ambas investigaciones encontraron datos alarmantes respecto a la práctica de este fenómeno pero sobretodo la presencia de consecuencias que lógicamente ponen en riesgo la integridad emocional, psicológica y física de los adolescentes.

\section{Conclusiones y recomendaciones}

Conclusiones: la prevalencia de sexting en los adolescentes de la Unidad Educativa Manuela Garaicoa de Calderón fue elevada, las dos terceras partes practicaron sexting pasivo y un tercio de ellos activo. La práctica general de sexting prevaleció en la adolescencia media, fue más frecuente en el sexo femenino, se registró mayor práctica en la residencia urbana y el bachillerato. El sexting pasivo se fundamentó en la recepción de fotos, seguido de mensajes de texto, y el medio más usado para dicha práctica fue Facebook. El sexting activo estuvo caracterizado por el envío de fotos o videos en ropa interior y de textos con proposiciones sobre actividad sexual por medio de un teléfono móvil. Esta práctica resultó ser obligada en aproximadamente un tercio de ellos, y trajo consecuencias en casi la mitad de los adolescentes siendo la principal consecuencia el Grooming.

Recomendaciones: resulta necesario implementar una orientación a los jóvenes respecto al manejo de estos elementos, sobre los beneficios, pero sobretodo los riesgos que implican. Además, es necesario realizar estudios similares al propuesto en otras instituciones de tal forma que se pueda tener un conocimiento más profundo del tema a nivel de ciudad, de la provincia y el país en los cuales se pueda incluso indagar las motivaciones que tienen los adolescentes para llevar a cabo esta práctica.

\section{Fuente de Financiamiento}

Este estudio es autofinanciado.

\section{Conflicto de Intereses}

No existen conflictos personales, profesionales, financieroso de otro tipo.

\section{Consentimiento Informado}

Los autores cuentan con el consentimiento informado de los pacientes para la investigación, la publicación del caso y sus imágenes. 


\section{Referencias Bibliográficas}

1. Ruiz J, Sánchez J, Trujillo J. Utilización de Internet y dependencia a teléfonos móviles en adolescentes. Revista Latinoamericana de Ciencias Sociales, Niñez y Juventud 2016, Vol 14 (2), pp. 1357-1369. Disponible en: http: //www.scielo.org.co/pdf/rlcs/v14n2/v14n2a33.pdf

2. La Red es usada para perpetrar nuevos delitos [Internet]. El Comercio. [citado 13 de octubre de 2019]. Disponible en: https://www.elcomercio.com/actualidad/internetsexting-pornografia-redessociales-delitos.html

3. Homayoun A. La vida secreta de los adolescentes en las redes sociales. The New York Times [Internet]. el 14 de junio de 2017; Disponible en: https://www.nytimes.com/ 18 . es/2017/06/14/redes-adolescentes-precauciones/

4. Las redes sociales son un arma de doble filo: Poniatowska - Grupo Milenio [Internet]. el 30 de Enero del 2015 [citado el 5 de noviembre de 2017]. Disponible en: http: //www.milenio.com/cultura/redes-sociales-arma-doblePoniatowska-honoris_causa_UCM_0_455354594.html

5. Los riesgos de Internet y las redes sociales para los 20 . niños [Internet]. guiainfantil.com. Disponible en: https: //www.guiainfantil.com/articulos/educacion/nuevastecnologias/internet-y-las-redes-sociales-riesgos-paralos-ninos/

6. ¿A qué edad deben tener un celular los niños? [Internet]. 2015 [citado 13 de octubre de 2019]. Disponible en: https://www.lanacion.com.ar/tecnologia/a-queedad-los-ninos-deben-tener-un-celular-nid1819253

7. ¿El primer móvil a los 10 años? [Internet]. La Vanguardia. 2016 [citado 13 de octubre de 2019]. Disponible en: https://www.lavanguardia.com/tecnologia/20160523/ 401986500346/estudio-primer-movil-internet-ninos.html

8. El 'sexting' se filtra en el Ecuador, advierten terapeutas familiares | Doctor Tecno | Vida y Estilo | El Universo [Internet]. [citado 13 de octubre de 2019]. Disponible en: https://www.eluniverso.com/vidaestilo/2015/11/22/nota/5253288/sexting-se-filtraecuador-advierten-terapeutas-familiares

9. Tipantuña K. Adicción a internet y sus consecuencias en adolescentes de la ciudad de Quito en el año 2013. Pontificia Universidad Católica del Ecuador. Facultad de Medicina. Quito, 2013. Disponible en: http://repositorio.puce. edu.ec/bitstream/handle/22000/5936/T-PUCE-6101.pdf

10. Gámez-Guadix M, de Santisteban P, Resett S. Sexting among Spanish adolescents: Prevalence and personality profiles. Psicothema. febrero de 2017;(29.1):29-34.

11. $20 \%$ de adolescentes practican «sexting» pese a riesgos [Internet]. El Comercio. [citado 13 de octubre de 2019]. Disponible en: https://www.elcomercio.com/tendencias/ adolescentes-practican-sexting-eeuu.html

12. Lounsbury K, Mitchell K, Finkelhor D. The True Prevalence of "Sexting". Crimes Against Children Research Center. University of Hampshire. 2011.

13. West JH, Lister CE, Hall PC, Crookston BT, Snow PR, Zvietcovich ME, et al. Sexting among peruvian adolescents. BMC Public Health. diciembre de 2014;14(1):811.
14. Reyns B, Henson B, Fisher B. Digital Deviance: Low Self-Control and Opportunity as Explanations of Sexting Among College Students. Sociological Spectrum. 17 de abril de 2014;34

5. McLaughlin, J. "Crime and Punisment: Teen Sexting in context" Penn State Law Review [Vol. 115:1] 5 de marzo del 2010.

16. Mejía-Soto G. Sexting: una modalidad cada vez más extendida de violencia sexual entre jóvenes. Perinatología y reproducción humana. diciembre de 2014;28(4):217-21.

17. Pastor EM, Gutiérrez JCS, Jiménez AG. Percepción de los riesgos en la red por los adolescentes en España: usos problemáticos y formas de control. :20.

18. King B. How Much Is Too Much? Limit Setting and Sexual Acting Out in a Digital Era. Journal of Clinical Psychology. 2012;68(11):1196-204.

19. Ringrose J, Gill R, Livingstone S, Harvey L. A qualitative study of children, young people and "sexting": a report prepared for the NSPCC [Internet]. 2012 [citado el 23 de noviembre de 2017].

20. El sexting activo entre adolescencias se asoció a una actividad más sexual [Internet]. News-Medical.net. 2014 [citado 13 de octubre de 2019]. Disponible en: https://www. news-medical.net/news/20141008/8459/Spanish.aspx

21. Agustina JR. ¿Menores infractores o víctimas de pornografía infantil? 2010;44.

22. Fajardo M, Gordillo M, Regalado A. Sexting: Nuevos Usos de la Tecnología y la Sexualidad en Adolescentes. International Journal of Developmental and Educational Psychology. INFAD Revista de Psicología, N¹-Vol.1, 2013 [citado el 23 de Noviembre del 2017]. pp:521-53. Disponible en: http://dehesa.unex.es/bitstream/handle/10662/958/02149877_2013_1_1_521.pdf?sequence=4\&isAllowed=y

23. Alonso P. Evaluación del fenómeno del Sexting y de los Riesgos emergentes de la Red en adolescentes de la Provincia de Ourense. Tesis Doctoral [internet]. Universidad de Vigo, 2017. Disponible en: http://www.investigo.biblioteca.uvigo.es/xmlui/ bitstream/handle/11093/786/Evaluaci\%C3\%B3n_del_ fen\%C3\%B3meno_del_sexting.pdf?sequence=1

Recibido: 9 de octubre de 2020

Aceptado: 22 de octubre de 2020 